\title{
The Efficacy of the Cook-Swartz Implantable Doppler in the Detection of Free-Flap Compromise: A Systematic Review and Meta-Analysis
}

\author{
Riaz A. Agha, BSc, MBBS, MSc, MRCS, FHEA, FRSPH ${ }^{1}$ Buket Gundogan, BSc ${ }^{2}$ \\ Alexander J. Fowler, BSc, MBBS ${ }^{3}$ Thomas W. H. Bragg, MBChB, FRCS ${ }^{4}$ Dennis P. Orgill, MD, PhD ${ }^{5}$
}

${ }^{1}$ Department of Plastic Surgery, Guy's and St. Thomas' NHS Foundation Trust, London, United Kingdom

2 University College London Medical School, London, United Kingdom

3 Department of Vascular Surgery, Guy's and St. Thomas' NHS

Foundation Trust, London, United Kingdom

${ }^{4}$ Department of Plastic Surgery, Stoke Mandeville Hospital, Aylesbury, Bucks, United Kingdom

${ }^{5}$ Division of Plastic Surgery, Brigham and Women's Hospital, Boston, Massachusetts and Harvard Medical School, Boston, Massachusetts

J Reconstr Microsurg Open 2016;1:73-81.

\author{
Address for correspondence Buket Gundogan, BSc, University \\ College, London Medical School, London, United Kingdom \\ (e-mail: buket.gundogan.11@ucl.ac.uk).
}

\author{
Abstract \\ Keywords \\ - Doppler \\ - Cook-Swartz Doppler \\ - free flap \\ - reconstruction \\ - microsurgery
}

Background Reducing free-flap failure rates is a key goal of any microsurgical unit. The Cook-Swartz implantable Doppler (CSD) can be used to monitor flap vascularity. We conducted a systematic review and meta-analysis to compare the efficacy of the CSD with clinical monitoring to prevent flap failure.

Methods A comprehensive literature search was performed using MEDLINE, EMBASE, PsycINFO, EBSCO, the Cochrane Library, CINAHL, SCOPUS, SciELO, NHS evidence, and online clinical trial registers from 1966 until December 31, 2015. Studies comparing flap failure rates in the CSD and clinically monitored groups were considered. Screening and data extraction was performed by two independent researchers.

Results Overall, eight articles met the inclusion criteria, involving 3,756 patients and 3,801 flaps. The average failure rate in the clinical group was 3.5\% and in the Doppler group was $2.0 \%$. A fixed effects meta-analysis was performed and found a reduced failure rate with the use of the CSD (odds ratio $=0.37$ [0.21-0.64], $p=0.0005$ ).

Conclusion Deployment of the CSD can lower flap failure rates and has the potential to be a useful adjunct to clinical monitoring of free flaps. Further research is needed to confirm its benefits and refine its indications to optimize cost-effectiveness.

\section{Background}

\section{Free-Flap Failure}

Free-flap reconstruction for large soft tissue defects is increasingly common, and plays an important role in the field of plastic and reconstructive surgery. ${ }^{1}$ Minimizing flap failure is a key goal for any microsurgical unit. Data suggest that the commonest cause of failure is a problem with the venous anastomosis, occurring within the first 24 to 48 hours postoperatively. ${ }^{2-4}$ This affects up to $20 \%$ of all free flap reconstructions, depending upon their location and entails significant physical, psychological, and emotional morbidity for patients. ${ }^{5-7}$

Impending flap failure necessitates a further general anesthetic and reoperation, potentially out of hours, which received

February 22, 2016 accepted after revision

May 26, 2016

published online

September 2, 2016
DOI http://dx.doi.org/ 10.1055/s-0036-1585086. ISSN 2377-0813.
Copyright $\odot 2016$ by Thieme Medical Publishers, Inc., 333 Seventh Avenue, New York, NY 10001, USA. Tel: +1(212) 584-4662.
License terms

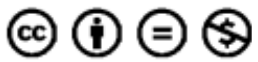


provides further logistical and practical challenges. ${ }^{7}$ Venous congestion may require the use of medicinal leeches. It is very rare that leeches can salvage larger-sized flaps, and can also be highly unpleasant for patients, predisposing them to infection and anemia. ${ }^{8,9}$

\section{Monitoring of Free Flaps and the Cook-Swartz Doppler} Early recognition of flap compromise is the primary aim of every microsurgical unit. Prompt intervention and rescue is critical to salvage and ensuring flap survival. However, the complexities of free flap microcirculation are often difficult to assess, and there is a wide array of monitoring modalities. ${ }^{10}$ The traditional method of monitoring is performed clinically and is based upon subjective clinical observations. These tests are performed typically on a "skin paddle" 11 and include assessment of color, capillary refill, temperature, turgor, and use of a handheld Doppler device. ${ }^{1}$

Unlike solid organ transplantation, there is no objective assessment-such as decreased urine output in renal transplantation. Currently, there are no suitable imaging modalities for assessing microvascular flow and specifically slow venous flow problems, reports in the literature on the use of nuclear medicine techniques are largely experimental. ${ }^{12}$ Early compromise, which entails the large majority of flap failure, is often asymptomatic. Skin changes can take a while to develop, potentially increasing ischemic times and reducing the possibility of successful salvage. ${ }^{13}$ Some free flaps are more challenging to monitor, such as muscle flaps, those with dark skin or buried flaps within the head and neck.

The Cook-Swartz implantable Doppler (CSD) monitors the arterial or venous blood velocity from free flaps. ${ }^{14}$ It is the only device currently on the market that allows such monitoring and is protected by patent. It consists of a $20 \mathrm{MHz}$ ultrasonic Doppler crystal, a silicone cuff and a monitoring unit. ${ }^{15,16}$ This cuff secures the Doppler to the flap's vein or artery at the time of the operation and is placed downstream of the microvascular anastomosis. ${ }^{16}$ The Doppler provides monitoring for 5 to 10 days postoperatively and is removed by simple traction. ${ }^{14}$ Several studies have shown that use of this device increases success and salvage rates as it provides an earlier warning when compared with clinical monitoring. ${ }^{11,17,18}$

\section{Why it is Important to do this Systematic Review}

To our knowledge, there have only been two systematic reviews involving the CSD. ${ }^{19,28}$ Since then further studies have been published and this area is an active research front.

\section{Objectives}

\section{Primary Objective}

To compare the efficacy of the CSD against clinical assessment (the traditional standard) in the detection of free-flap compromise.

\section{Secondary Objectives}

To determine the absolute indications for use of the Doppler (if any). Quantify positive and negative predictive values as well complications associated with the use of the CSD.

\section{Methods}

This systematic review was conducted in accordance with the Cochrane handbook for systematic reviews and reported in line with the preferred reporting items for systematic reviews and meta-analyses (PRISMA) statement. ${ }^{20} \mathrm{~A}$ protocol was

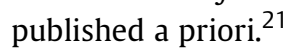

\section{Inclusion and Exclusion Criteria}

Types of Studies, Participants, Interventions, and Comparators

Studies comparing the use of the CSD with clinical assessment in detecting impending free-flap failure were included. Any article where the data were duplicated was excluded, as were articles not describing original data; such as editorials, letters, commentaries, and discussion pieces. There were no limitations on location of the flap or technique utilized.

\section{Types of Outcome Measures}

\section{Primary Outcome}

Flap failure rate, defined as the number of free flaps lost divided by the total number of flaps performed.

\section{Secondary Outcomes}

Positive predictive value (PPV) and negative predictive values, time to detection will be reported where possible and compared between CSD and clinical groups. Any complications associated with CSD use will be described.

\section{Search Methods for Identification of Studies}

\section{Electronic Searches}

The following electronic databases were searched from 1966 until December 31, 2015: MEDLINE, EMBASE, PsycINFO, EBSCO, the Cochrane Library, CINAHL, SCOPUS, SciELO, NHS evidence, uptodate.com, clinicaltrials.gov, WHO meta-register of controlled trials (available at: who.int/ictrp/en), and controlled-trials.com.

\section{Search Terms and Keywords}

The search strategy was developed to locate articles related specifically to the CSD and provide evidence for the objectives previously stated. The search used the English language keywords combined with Boolean logical operators. The following terms were utilized without any limits: "Implantable doppler" OR "Cook-Swartz implantable doppler" OR "Cook-Swartz Implantable doppler" The search was not limited by language. Any non-English articles identified had their title and abstract screened and the full text was obtained if required. If full text was not available, then the authors were contacted to obtain an English language copy of the full text.

\section{Other Resources}

A hand search of the references of articles located by the search strategy was used to identify any additional relevant studies. 


\section{Selection of Studies}

Studies identified by the electronic and manual search strategies were entered into a database. Results including citation, title, and abstracts were populated into EndNote (Thomson Reuters, New York, NY) and duplicates removed. Titles and abstracts were screened by two authors (B. G. and A. J. F.), any conflicts not resolvable between the two were referred to the lead author (R. A. A.) for resolution. Articles selected after title and abstract screen had their full text downloaded and a further assessment was made. Once the articles were selected for inclusion, data extraction took place.

\section{Data Extraction and Management}

Data were extracted independently by two authors (B. G. and A. J. F.) utilizing a standard extraction form where all data for each study were collated. Any conflicts were resolved by discussion; where resolution wasn't possible, the lead author (R. A. A.) made the final decision. These data were then entered into a Microsoft Excel 2011 database (Microsoft, Redmond, WA). Data collected constituted three main areas:

1. Article information
a. Title
b. Authors
c. Year of publication
d. Journal

2. Characteristics

a. Setting and location of the study

b. Number of patients

c. Range of patients age

d. Flap types included

e. Clinical method of assessment

f. Doppler method of assessment (arterial vs. venous)

3. Results (divided clinical and Doppler)

1. Number of patients per group

2. Specific flaps in groups (where applicable)

3. Detected

4. Flap Salvage

5. Needless theater return

6. Complications

7. Lost flaps without any signs

8. Flaps correctly monitored

\section{Assessment of Study Quality and Bias in Included Studies}

Quality of evidence was assessed using the Grading of Recommendation Assessment, Development and Evaluation (GRADE) system as proposed by Balshem et al. ${ }^{22}$ Any information missing from the studies was documented and assessed to ascertain the risk of incomplete statistical sets.

\section{Measures of Treatment Effect}

The treatment effect was measured using an odds ratio for the studies, since the data were dichotomous (event vs. no event, where an event is a correctly identified flap necrosis or a true positive).

\section{Dealing with Missing Data}

Where data from studies were missing, the original investigators were contacted to request missing data. If this was not feasible, available data were analyzed and the potential impact of this is addressed in the discussion.

\section{Assessment of Heterogeneity}

Heterogeneity between studies was assessed using Higgins and Thompson $\mathrm{I}^{2}$, which measures the percentage variability in results attributable to heterogeneity between studies rather than sampling error. ${ }^{23}$ Variability in the intervention effects in studies was tested for statistical heterogeneity utilizing Tau-squared $\left(\mathrm{T}^{2}\right), \mathrm{I}^{2}$, and chi-squared $\left(\chi^{2}\right)$ with corresponding $p$ values calculated; the Cochrane tests.

The value of $\chi^{2}$ statistics in the forest plot presents the assessment of whether the differences in results are compatible with chance alone. A large value of $\chi^{2}$ test relative to its degree of freedom or a low $p$ value indicates statistical variation (heterogeneity) beyond chance.

The $\mathrm{I}^{2}$ percentage was interpreted as follows:

1. 0 to $30 \%$ may not be important

2. 30 to $60 \%$ may represent moderate heterogeneity ${ }^{\mathrm{a}}$

3. 50 to $90 \%$ may represent substantial heterogeneity ${ }^{\mathrm{a}}$

4. 75 to $100 \%$ represents considerable heterogeneity ${ }^{\mathrm{a}}$

Generation of statistical heterogeneity can be a consequence of clinical (participants, interventions, and outcomes) and/or methodological (study design and risk of bias) diversity or due to random error (chance) alone. $\mathrm{T}^{2}$ represents the estimated standard deviation of underlying effects across studies. The exact model utilized for metaanalysis was based upon the level of heterogeneity within our data; with a random effects model used if substantial or considerable and a fixed-effects analysis if moderate, respectively.

\section{Assessment of Reporting Biases}

To ascertain if studies with negative outcomes are not being published ("publication bias"), we visually assessed funnel plot asymmetry. ${ }^{23,24}$ If both positive and negative results are published, the plot should resemble a symmetrical, inverted funnel. The precision of the estimated intervention effect increases as the size of the sample included in the study increases. Smaller studies therefore scatter widely at the bottom, with larger, more powerful studies grouping more narrowly at the top.

\section{Data Synthesis and Statistical Analysis}

Outcomes were tabulated, with descriptive statistics performed as appropriate. Similarly, the detection rate of each modality was compared and synthesized where possible. Synthesis was performed utilizing Review Manager (RevMan [Computer program]. Version 5.3. Copenhagen: The Nordic Cochrane Centre, The Cochrane Collaboration, 2014) and an

\footnotetext{
a The importance of the observed $\mathrm{I}^{2}$ value depends on; magnitude and direction of effects and strength of evidence for heterogeneity such as $p$ value from $\chi^{2}$ or a confidence interval for $\mathrm{I}^{2}$.
} 
assessment of heterogeneity was made. Based upon this a diagnostic test accuracy and review of a meta-analysis was performed comparing CSD to clinical monitoring using good quality observational studies.

Rate of flap salvage was compared between modalities of monitoring to establish any correlation. The false positive and negative rates of the CSD were calculated.

\section{Subgroup Analysis}

Subgroup analyses were planned in our protocol as follows:

1. Different flap type (if $>3$ studies describing specific flap types)

2. Venous versus Arterial CSD probe placement

3. Different anatomic locations (if $>3$ studies describe the same anatomic locations)

However, as insufficient data were obtained, these subgroup analyses were not performed.

\section{Results}

\section{Results of the Search}

Following the electronic database search, 655 records were retrieved. After removing duplicates 251 records remained. The titles and abstracts of these studies were screened and 61 full-text articles were assessed for eligibility. Eight articles were deemed eligible for inclusion in the review. A summary of this process is displayed in -Fig. 1.

\section{Included Studies}

Eight articles were included in the systematic review, involving 3,756 patients and 3,801 flaps. The average age of study participants was 48.1 years (though two did not report this) 25 and all but one of the studies attached the CSD distal to the venous anastomosis alone. The level of evidence as assessed by GRADE scoring was low or moderate for the included studies. ${ }^{22}$ A summary of included studies is available in -Table 1.

\section{Effects of the Cook-Swartz Doppler}

\section{Primary Outcome}

Flap failure rate was calculated for both CSD and clinically monitored flaps (-Table 2). The Ho et $\mathrm{al}^{26}$ article was excluded, since it compared the CSD on high risk cases versus clinical monitoring on low cases. Frost et $\mathrm{al}^{27}$ was excluded from qualitative synthesis since both the CSD and clinical monitoring groups also used microdialysis as an additional assessment and there was overlap between assessment methods in all groups.
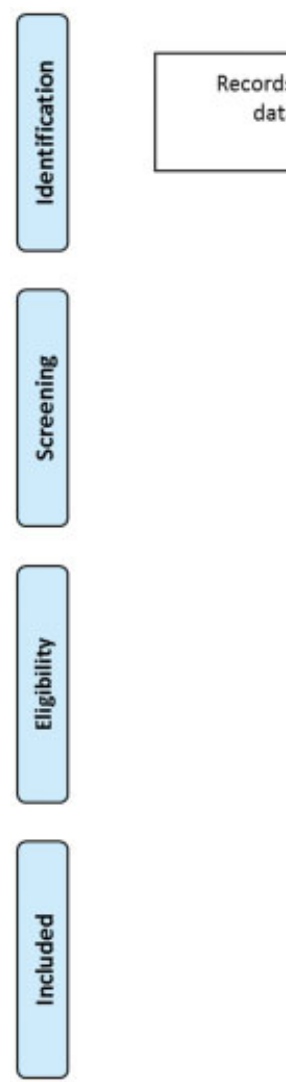

cords identified through
database searching

$$
(n=655)
$$
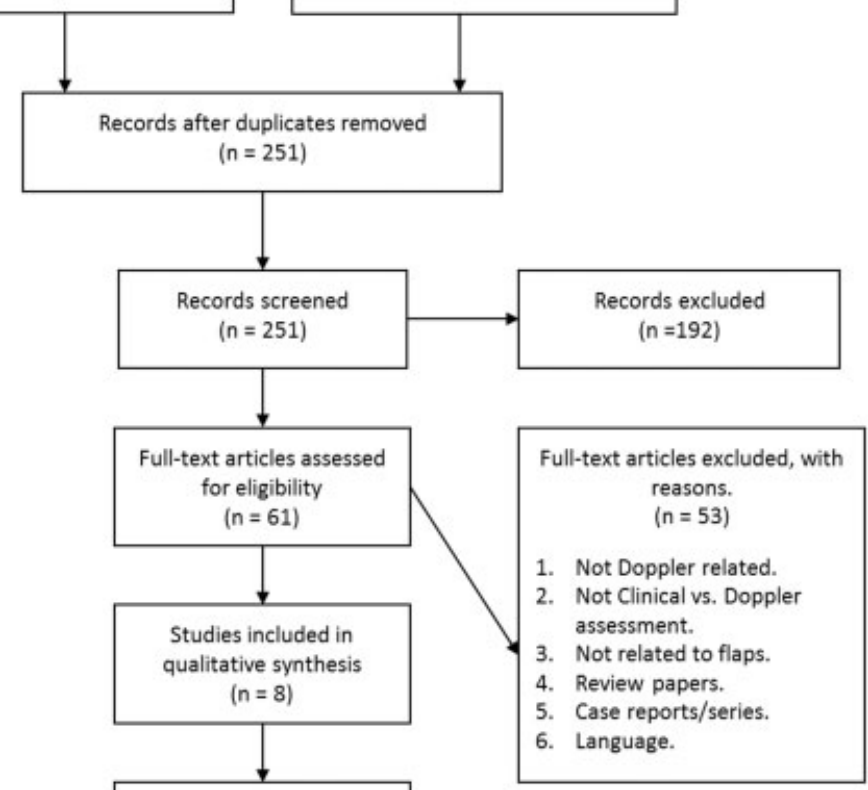

Studies included in

quantitative synthesis

(meta-analysis)

$$
(n=8)
$$

Fig. 1 PRISMA flow diagram of article selection. PRISMA, preferred reporting items for systematic reviews and meta-analyses. (Reprinted with permission from Moher et al. ${ }^{20}$ ) 


\begin{tabular}{|c|c|c|c|c|c|c|c|c|}
\hline 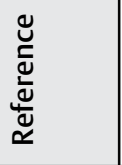 & & ஸ & $\oiiint$ & $=$ & \pm & $\sim$ & $\stackrel{\sim}{\sim}$ & $\AA$ \\
\hline 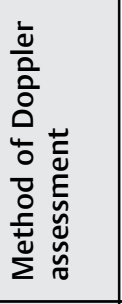 & 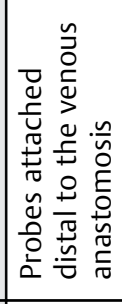 & 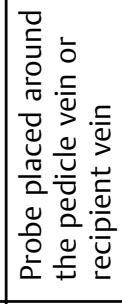 & 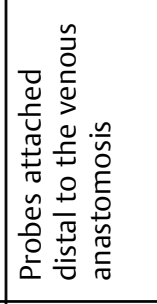 & 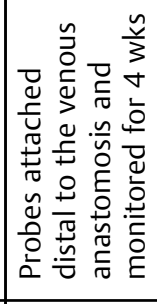 & 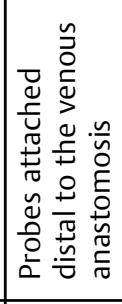 & 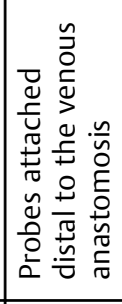 & 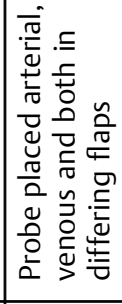 & 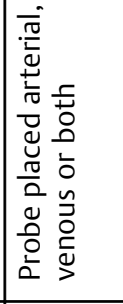 \\
\hline 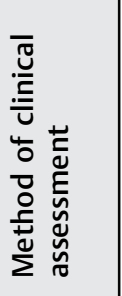 & $\frac{o}{z}$ & $\frac{\alpha}{z}$ & 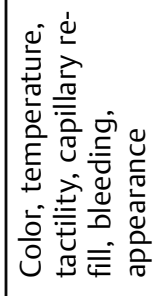 & 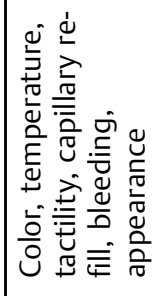 & 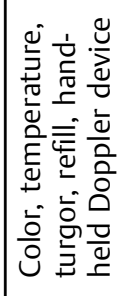 & 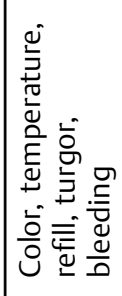 & $\frac{\alpha}{2}$ & 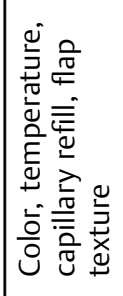 \\
\hline 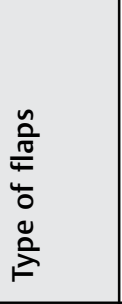 & 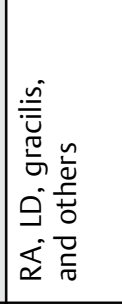 & 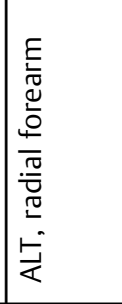 & 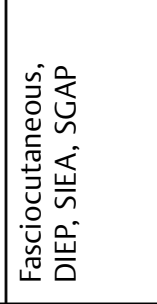 & 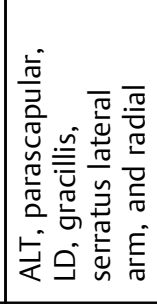 & 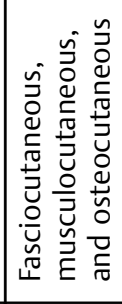 & $\frac{2}{z}$ & 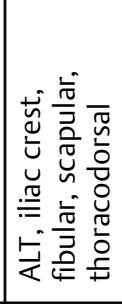 & 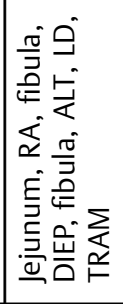 \\
\hline 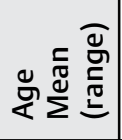 & 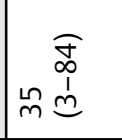 & $\frac{\alpha}{z}$ & 妾 & 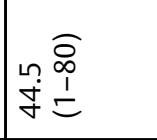 & 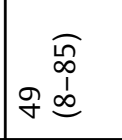 & 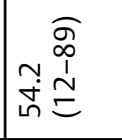 & 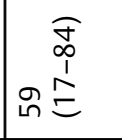 & 点 \\
\hline 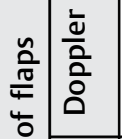 & 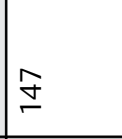 & $\stackrel{\bullet}{\bullet}$ & 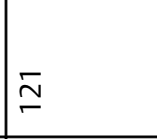 & $\stackrel{\curvearrowright}{\sim}$ & $\stackrel{\tilde{N}}{m}$ & $\stackrel{\mathscr{\leftrightarrow}}{\sim}$ & in & $\bar{N}$ \\
\hline 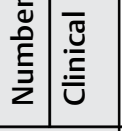 & $\stackrel{\stackrel{r}{m}}{\stackrel{m}{r}}$ & in & $\stackrel{\mathscr{\sim}}{\mathscr{\sigma}}$ & $\stackrel{2}{2}$ & 愈 & 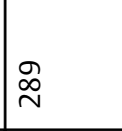 & {$\left[\begin{array}{l}\infty \\
m \\
m\end{array}\right.$} & $\stackrel{\infty}{\leftarrow}$ \\
\hline 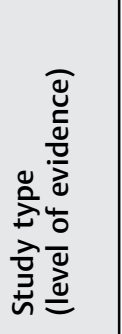 & 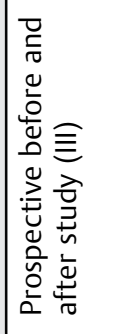 & 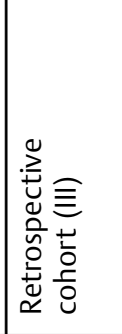 & 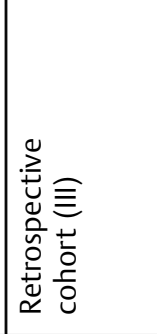 & 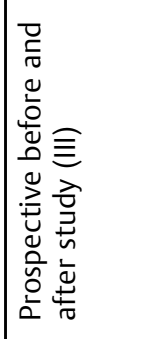 & 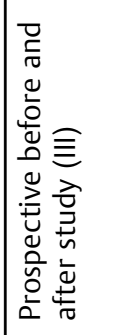 & 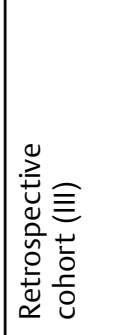 & 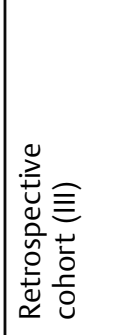 & 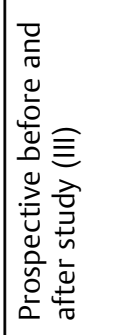 \\
\hline 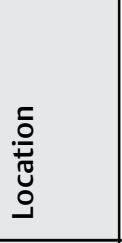 & $\frac{o}{z}$ & 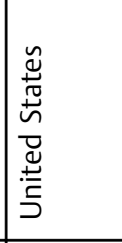 & 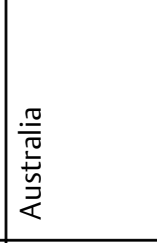 & 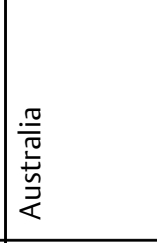 & 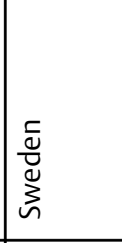 & 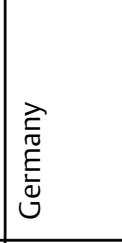 & 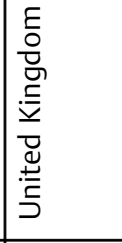 & $\frac{\alpha}{z}$ \\
\hline 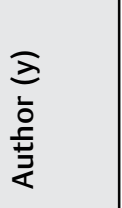 & 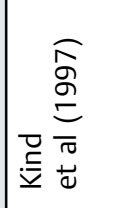 & 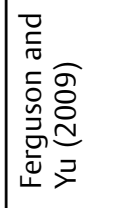 & 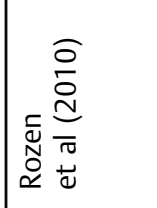 & 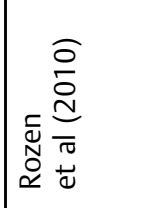 & 告 & 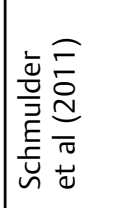 & 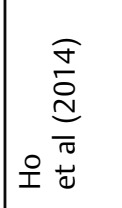 & 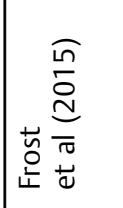 \\
\hline
\end{tabular}


Table 2 Flap failure rates of clinical and Doppler monitoring, as a percentage

\begin{tabular}{|l|l|l|}
\hline Study (y) & Clinical flap failure rate (\%) & CSD flap failure rate (\%) \\
\hline Kind et al $(1998)^{18}$ & $3.11 \%(41 / 1,317)$ & $0 \%(0 / 147)$ \\
\hline Ferguson and Yu $(2009)^{25}$ & $0 \%(0 / 59)$ & $6.25 \%(1 / 16)$ \\
\hline Rozen et al $(2010)^{16}$ & $4.00 \%(18 / 426)$ & $1.65 \%(2 / 121)$ \\
\hline Rozen et al $(2010)^{11}$ & $15.0 \%(3 / 20)$ & $0 \%(0 / 20)$ \\
\hline Smit et al $(2010)^{10}$ & $0.33 \%(1 / 307)$ & $0.62 \%(2 / 323)$ \\
\hline Schmulder et al $(2011)^{2}$ & $10.7 \%(31 / 289)$ & $3.86 \%(10 / 259)$ \\
\hline Overall failure rate & $3.5 \%(96 / 2,742)$ & $2.0 \%(19 / 945)$ \\
\hline
\end{tabular}

Abbreviation: CSD, Cook-Swartz implantable Doppler.

\section{Meta-Analysis}

A meta-analysis was performed using a random effects model as heterogeneity was substantial with $\mathrm{I}^{2}=71 \%$ (-Fig. 2), this showed no significant difference between the two groups $(p=0.87)$.

\section{Sensitivity Analysis}

When the article of Ho et $\mathrm{al}^{26}$ was excluded, then heterogeneity was low $\left(\mathrm{I}^{2}=33 \%\right)$ and a fixed-effects analysis showed there was a significant reduction in flap failure rate with CSD use (odds ratio $=0.37,95 \%$ confidence interval $=0.21-0.64$, $p=0.0005)$ as seen in - Fig. 3 below.

\section{Secondary Outcomes}

The sensitivity of clinical monitoring was calculated for six studies (-Table 3). The PPVs for CSD monitoring were estimable for seven of eight studies. These values ranged from 66.7 to $100 \%$. As for the clinical monitoring group, Rozen et $\mathrm{al}^{11}$ had a negative predictive value of $100 \%$ and two PPVs were calculated as 0.71 and 0.58 . $^{2,18}$

PPVs are shown in the table below for CSD only as there was insufficient data to calculate this for the clinical group (-Table 4).

\section{Time to Detection}

Four of the eight studies discussed, time to detection of flap compromise between clinical and CSD monitoring. Smit et al reported that the alteration of CSD signal was either before or at the same time as any clinical signs of flap compromise. ${ }^{14}$ Likewise, the study of Schmulder et al demonstrated that there was a statistically significant relationship between flap outcomes and the time of discovering flow compromise between CSD and clinical monitoring, where many re-explorations in the CSD group were performed within the first 48 hours postoperatively, earlier than the clinical group. ${ }^{2}$

Rozen et al noted that CSD use detected anastomotic insufficiency at an earlier time course than clinical monitoring in their study, with detection occurring many hours before clinical signs were evident. ${ }^{11}$ Frost et al demonstrated that CSD was able to detect changes in the flap a mean of 97.5 minutes earlier than clinical assessment. ${ }^{27}$

Complications were found to be infection and anastomotic insufficiency. ${ }^{11}$ All other studies reported no complications related to CSD use.

\section{Assessment of Reporting Biases}

A funnel plot was generated, but with less than 10 studies, appropriate interpretation was not possible.

\section{Discussion}

Our analysis of over 3,800 flaps showed that flap monitoring with the CSD was superior to clinical monitoring when assessing flap failure rates (2.0 vs. 3.5\%, respectively). The CSD was deployed across a wide range of settings including; head and neck, breast and limb reconstruction, and a wide variety of flaps types including; musculocutaneous, fasciocutaneous, and buried flaps. The PPV of the CSD was highly variable from 66.7 to $100 \%$ but negative predictive values were 90.9 to $100 \%$. Frost et al was not included in our meta-

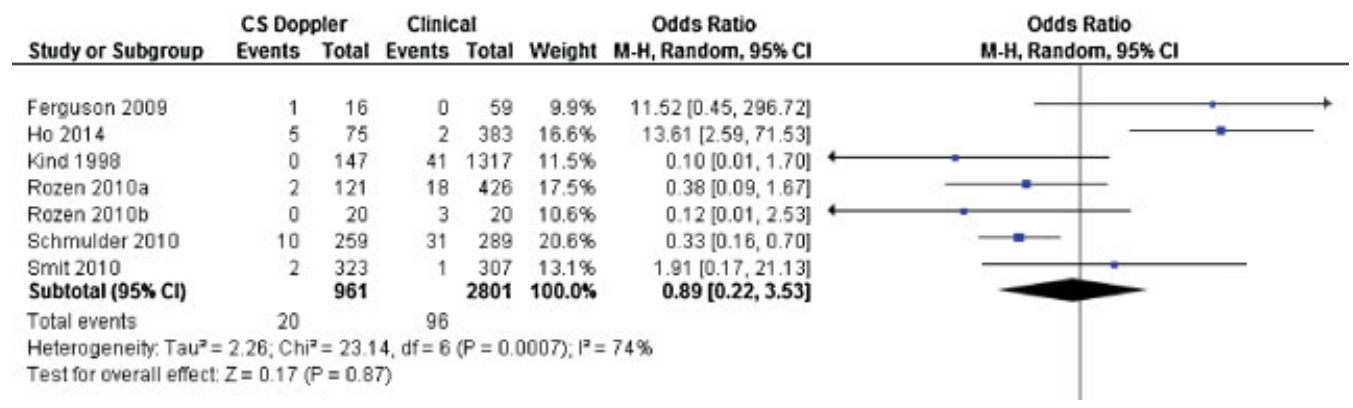

Fig. 2 Forest plot of studies included in the systematic review. 


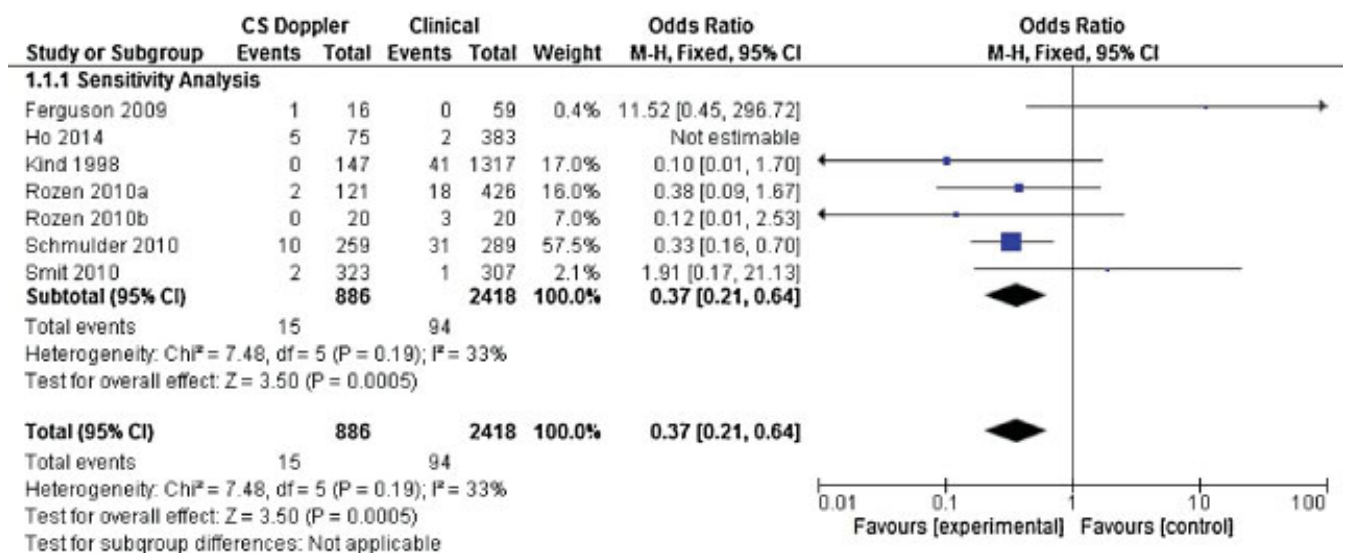

Fig. 3 Forest plot of studies in the sensitivity analysis.

analysis as the flaps in the study overlapped in their assessment methods. CSD, clinical and microdialysis was employed for the same flaps. ${ }^{27}$ For these reasons, an accurate comparison between CSD and clinical assessments could not be made.

No studies specifically reported using the CSD in Fitzpatrick skin types 5 and 6 , where traditional clinical monitoring techniques may be more difficult. The time critical nature of flap loss is clear. Schmulder et al found that the mean time to discovery of flap compromise was 1.333 days in the CSD group versus 2.423 days in the clinically monitored group. Linked to this, the same study reported a higher successful reexploration rate within and after 48 hours together with a lower failure rate in the CSD group. ${ }^{2}$ However, the variability with which this was reported and the data provided precluded any attempts at data synthesis.

Overall, the quality of evidence available for synthesis was moderate or low, as assessed by GRADE and there were no randomized controlled trials (RCTs). One group undertook a prospective before and after study (Rozen et al) looking specifically at lower limb defects, however, with only 20 patients in each group, the findings of this study are more prone to bias than the larger studies, especially since a power calculation was not performed a priori. ${ }^{11}$ However, this was also the only study amongst the six to undertake a baseline prognostic comparison of the two groups to assess for significant differences in; sex, age, smoking status, corticosteroid use, hypertension, diabetes mellitus, and mean ischemic time. Despite the best efforts to control two groups of patients for type of flap and type of patient, there could be anatomical confounders between the two groups.

\section{Strengths of this Review}

This was a carefully planned systematic review. Our group has performed several systematic reviews previously and has developed some expertise in this area. This is the first systematic review to look at the CSD. We asked a focused question and implemented strict inclusion and exclusion criteria to ensure only relevant articles of sufficient quality were included. Article selection and data extraction were performed independently by two researchers, and all decisions were finally authorized by the lead author (R. A. A.). We have reported our study in line with the PRISMA criteria and took every effort to ensure the review was performed with impartiality and minimum bias. Our selection of a binary outcome for our primary analysis (flap failure) nullifies issues often found in systematic reviews, such as the heterogeneous nature of outcomes and associated observer bias potentially confounding results. In addition, the problem with comparing PPVs for clinical and CSD-based monitoring is that the former is inevitably going to be more accurate-if one waits long

Table 3 The sensitivity of clinical and CSD monitoring for each included study

\begin{tabular}{|l|l|l|l|l|}
\hline \multirow{2}{*}{ Study (y) } & Clinical & \multicolumn{2}{l|}{ CSD } \\
\cline { 2 - 5 } & False-positive & False-negative & False-positive & False-negative \\
\hline Kind et al $(1998)^{18}$ & $0 \%(0 / 1,317)$ & $0 \%(0 / 1,317)$ & $2.7 \%(4 / 147)$ & $0 \%(0 / 147)$ \\
\hline Ferguson and Yu $(2009)^{25}$ & $0 \%(0 / 59)$ & $0 \%(0 / 59)$ & $31 \%(5 / 16)$ & $9.1 \%(1 / 11)$ \\
\hline Rozen et al $(2010)^{16}$ & $0 \%(0 / 374)$ & $1.6 \%(6 / 374)$ & $9.1 \%(1 / 11)$ & $0 \%(0 / 110)$ \\
\hline Rozen et al (2010) & $0 \%(0 / 20)$ & $0 \%(0 / 20)$ & $0 \%(0 / 20)$ & $0 \%(0 / 20)$ \\
\hline Smit et al (2010) & 10 & $\mathrm{~N} / \mathrm{R}$ & $\mathrm{N} / \mathrm{R}$ & $\mathrm{N} / \mathrm{R}$ \\
\hline Schmulder et al (2011) & $\mathrm{N} / \mathrm{R}$ & $\mathrm{N} / \mathrm{R}$ & $8.3 \%(3 / 36)$ & $\mathrm{N} / \mathrm{R}$ \\
\hline Ho et al $(2014)^{27}$ & $0 \%(0 / 26)$ & $\mathrm{N} / \mathrm{R}$ & $33.3 \%(3 / 9)$ & $4.8 \%(3 / 62)$ \\
\hline Frost et al $(2015)^{26}$ & $\mathrm{~N} / \mathrm{R}$ & $3 / 18$ & $0 / 21$ & $1 / 21$ \\
\hline
\end{tabular}

Abbreviations: CSD, Cook-Swartz implantable Doppler; N/R, not reported. 
Table 4 Positive and negative predictive values for the CSD

\begin{tabular}{|c|c|c|}
\hline Study (y) & Positive predictive value (\%) & Negative predictive value (\%) \\
\hline Kind et al $(1998)^{18}$ & 97.3 & 100 \\
\hline Ferguson and $\mathrm{Yu}(2009)^{25}$ & 69 & 90.9 \\
\hline Rozen et al $(2010)^{16}$ & 90.9 & 100 \\
\hline Rozen et al $(2010)^{11}$ & 100 & 100 \\
\hline Smit et al $(2010)^{10}$ & $N / R$ & $N / R$ \\
\hline Schmulder et al $(2011)^{2}$ & 91.7 & $N / R$ \\
\hline Ho et al $(2014)^{27}$ & 66.7 & 95.2 \\
\hline Frost et al $(2015)^{26}$ & 100 & 95 \\
\hline
\end{tabular}

Abbreviations: CSD, Cook-Swartz implantable Doppler; N/R, not reported.

enough, failure becomes clinically obvious. Earlier detection is likely to have played a key role in the difference in flap failure rates between the two groups.

\section{Limitations}

Despite our best efforts to locate appropriate studies, several potentially important studies could not be included as full texts were not available. Authors were contacted to try and access these, but we received no response. Moreover, the included studies did not account for the learning curve associated with the use of the CSD and with the use of venous couplers. We were also looking at studies comparing either clinical monitoring or CSD, not CSD in conjunction with clinical monitoring, which may provide optimal results.

We comprehensively reviewed the available literature, including trial databases. However, no RCTs were available for inclusion in our study. The lack of data from the highest quality study methodology means a careful assessment of bias has to be undertaken.

The reporting of flap failure and adequate detection of flap compromise is complex with current reporting methods; however the flow diagram utilized by Rozen et al serves as a potentially useful template for future work. ${ }^{16}$ The free flap failure rates were very low across all studies which may mean that only technically excellent high volume centers/surgeons are reporting their results, limiting external validity.

\section{Cost-Effectiveness}

If there is indeed a true benefit (subject to confirmation in a high-quality RCT or adequately powered prospective cohort studies), it is likely the CSD would be best deployed in high risk cases. Ho et al followed such a strategy, deploying the CSD in reoperations, those who had received radiotherapy, buried flaps, muscle flaps, those with very pale or very dark complexions, where there was difficult access for clinical monitoring or where there had been difficulties with intraoperative perfusion of the flap. ${ }^{26}$ We can add significant comorbidities such as peripheral vascular disease and diabetes to this list, as well as smokers and surgeon choice-where their experience, intraoperative events or anatomical considerations necessitate closer adjunctive monitoring in addition to the routine clinical monitoring.

The cost of the CSD is U.S. $\$ 3,000$ for the reusable base station and U.S. $\$ 300$ for the disposable probes. With the clinical monitored group having an overall success rate of $96.5 \%$, adding this marginal cost to every case may not be cost-effective but to a certain extent this depends on the balance of costs and reimbursements in the relevant health care system. Notwithstanding this, adding this marginal cost to the higher risk cases, as defined above, where flap failure is relatively more frequent is certainly a logical position. This strategy is supported by Rozen et al's study where they calculated the cost to salvage one flap at $\$ 20,000$. $^{11}$

\section{Future Directions}

Further research is now required to determine the actual benefit of the CSD and the associated learning curve in its use. Ideally this would be in the format of a pragmatically designed RCT-at present there is equipoise for such a study. The use of the CSD may be highly beneficial in cross-specialty flaps, where patients may not be monitored by staff accustomed to free flaps, for example, where a patient returns to a nonplastic surgical ward after free flap surgery. A large observational cohort study, where specific flap types can be assessed with an appropriately powered cohort may well demonstrate important findings. A registry-based design may also be useful.

The other benefits of the CSD need to be quantified too if possible, such as the value of monitoring without waking the patient up at night or without having to use a mirror or flexible fiber optic to monitor a buried flap. This has to be weighed up against the more variable PPV-necessitating clinical correlation, hence its role is as an adjunct to clinical monitoring, not a replacement for it. We also call for further research to assess its cost-effectiveness.

\section{Conclusion}

Deployment of the CSD can lower flap failure rates and has the potential to be a useful adjunct to clinical monitoring of free flaps. Further research is needed to confirm its benefits and refine its indications to optimize cost-effectiveness.

\section{Author Contributions}

Lead author R. A. A. conceived and designed the review. T. B. and D. O. contributed to the concept. R. A. A. and A. J. F. coordinated the review, designed search strategies. B. G. 
and A. J. F performed data collection for the review, undertook searches, screened search results, organized retrieval of articles, screened retrieved articles against eligibility criteria, appraised quality of articles and extracted data from articles. B. G. entered data into RevMan and analyzed data. B. G., A. J. F. and R. A. A. interpreted the data. All authors contributed to the writing of the article and its critical revision.

\section{Protocol Registration}

PROSPERO: National Institute of Health Research (NIHR) Prospective Register of Systematic Reviews CRD42013005818.

\section{Conflict of Interest}

None.

\section{Acknowledgments}

The authors thank the UCL Medical School Royal Free library staff with particular mention to Angela Young and Ruth Muscat for their training and support. Support for the project (DPO) was provided by the Gillian Reny Stepping Strong Fund.

\section{References}

1 Jallali N, Ridha $\mathrm{H}$, Butler PE. Postoperative monitoring of free flaps in UK plastic surgery units. Microsurgery 2005;25(6):469-472

2 Schmulder A, Gur E, Zaretski A. Eight-year experience of the CookSwartz Doppler in free-flap operations: microsurgical and reexploration results with regard to a wide spectrum of surgeries. Microsurgery 2011;31(1):1-6

3 Liu Y, Zhao YF, Huang JT, et al. Analysis of 13 cases of venous compromise in 178 radial forearm free flaps for intraoral reconstruction. Int J Oral Maxillofac Surg 2012;41(4):448-452

4 Hidalgo DA, Jones CS. The role of emergent exploration in freetissue transfer: a review of 150 consecutive cases. Plast Reconstr Surg 1990;86(3):492-498, discussion 499-501

5 Ali R, Bernier C, Lin YT, et al. Surgical strategies to salvage the venous compromised deep inferior epigastric perforator flap. Ann Plast Surg 2010;65(4):398-406

6 Yu P, Chang DW, Miller MJ, Reece G, Robb GL. Analysis of 49 cases of flap compromise in 1310 free flaps for head and neck reconstruction. Head Neck 2009;31(1):45-51

7 Chen KT, Mardini S, Wei FC. Intraoperative vascular findings predict salvage outcome in free flaps with circulatory compromise. J Reconstr Microsurg 2006;22(07):A020

8 Utley DS, Koch RJ, Goode RL. The failing flap in facial plastic and reconstructive surgery: role of the medicinal leech. Laryngoscope 1998;108(8 Pt 1):1129-1135

9 Bibbo C, Fritsche T, Stemper M, Hall M. Flap infection associated with medicinal leeches in reconstructive surgery: two new drugresistant organisms. J Reconstr Microsurg 2013;29(7):457-460

10 Smit JM, Zeebregts CJ, Acosta R, Werker PM. Advancements in free flap monitoring in the last decade: a critical review. Plast Reconstr Surg 2010;125(1):177-185

11 Rozen WM, Enajat M, Whitaker IS, Lindkvist U, Audolfsson T, Acosta R. Postoperative monitoring of lower limb free flaps with the Cook-Swartz implantable Doppler probe: A clinical trial. Microsurgery 2010;30(5):354-360

12 Dragu A, Linke R, Kuwert $\mathrm{T}$, et al. Interesting image. Tc-99m sestamibi SPECT/CT as a new tool for monitoring perfusion and viability of buried perforator based free flaps in breast reconstruction after breast cancer. Clin Nucl Med 2010;35(1):36-37

13 Lin SJ, Nguyen MD, Chen C, et al. Tissue oximetry monitoring in microsurgical breast reconstruction decreases flap loss and improves rate of flap salvage. Plast Reconstr Surg 2011;127(3): 1080-1085

14 Smit JM, Whitaker IS, Liss AG, Audolfsson T, Kildal M, Acosta R. Post operative monitoring of microvascular breast reconstructions using the implantable Cook-Swartz doppler system: a study of 145 probes \& technical discussion. J Plast Reconstr Aesthet Surg 2009;62(10):1286-1292

15 Swartz WM, Jones NF, Cherup L, Klein A. Direct monitoring of microvascular anastomoses with the 20-MHz ultrasonic Doppler probe: an experimental and clinical study. Plast Reconstr Surg 1988;81(2):149-161

16 Rozen WM, Chubb D, Whitaker IS, Acosta R. The efficacy of postoperative monitoring: a single surgeon comparison of clinical monitoring and the implantable Doppler probe in 547 consecutive free flaps. Microsurgery 2010;30(2):105-110

17 Paydar KZ, Hansen SL, Chang DS, Hoffman WY, Leon P. Implantable venous Doppler monitoring in head and neck free flap reconstruction increases the salvage rate. Plast Reconstr Surg 2010;125(4): $1129-1134$

18 Kind GM, Buntic RF, Buncke GM, Cooper TM, Siko PP, Buncke HJ Jr. The effect of an implantable Doppler probe on the salvage of microvascular tissue transplants. Plast Reconstr Surg 1998;101(5): 1268-1273, discussion 1274-1275

19 Poder TG, Fortier PH. Implantable Doppler in monitoring free flaps: a cost-effectiveness analysis based on a systematic review of the literature. Eur Ann Otorhinolaryngol Head Neck Dis 2013; 130(2):79-85

20 Moher D, Liberati A, Tetzlaff J, Altman DG; PRISMA Group. Preferred reporting items for systematic reviews and meta-analyses: the PRISMA statement. J Clin Epidemiol 2009;62(10): 1006-1012

21 Agha RA, Gundogan B, Fowler AJ, Bragg TW, Orgill DP. The efficacy of the Cook-Swartz implantable Doppler in the detection of freeflap compromise: a systematic review protocol. BMJ Open 2014; 4(3): 0004253

22 Balshem H, Helfand M, Schünemann HJ, et al. GRADE guidelines: 3. Rating the quality of evidence. J Clin Epidemiol 2011;64(4): 401-406

23 Higgins JPT, Green S. Cochrane Handbook for Systematic Reviews of Interventions Version 5.1.0 [updated March 2011]. The Cochrane Collaboration, 2011. Available at: www.cochrane-handbook.org. Accessed January 26, 2014

24 Sterne JA, Sutton AJ, Ioannidis JP, et al. Recommendations for examining and interpreting funnel plot asymmetry in metaanalyses of randomised controlled trials. BMJ 2011;343:d4002

25 Ferguson RE Jr, Yu P. Techniques of monitoring buried fasciocutaneous free flaps. Plast Reconstr Surg 2009;123(2):525-532

26 Frost MW, Niumsawatt V, Rozen WM, Eschen GE, Damsgaard TE, Kiil BJ. Direct comparison of postoperative monitoring of free flaps with microdialysis, implantable cook-swartz Doppler probe, and clinical monitoring in 20 consecutive patients. Microsurgery 2015;35(4):262-271

27 Ho MW, Cassidy C, Brown JS, Shaw RJ, Bekiroglu F, Rogers SN. Rationale for the use of the implantable Doppler probe based on 7 years' experience. Br J Oral Maxillofac Surg 2014;52(6): $530-534$

28 Han ZF, Guo LL, Liu LB, et al. A comparison of the Cook-Swartz Doppler with conventional clinical methods for free flap monitoring: A systematic review and a meta-analysis. 21;32:109-115 